\title{
Interplay between intramolecular and intermolecular structures of 1,1,2,2-tetrachloro-1,2-difluoroethane
}

\author{
M. Rovira-Esteva, ${ }^{1}$ N. A. Murugan, ${ }^{1}$ L. C. Pardo, ${ }^{1}$ S. Busch, ${ }^{2}$ J. Ll. Tamarit, ${ }^{1}$ Sz. Pothoczki, ${ }^{1}$ \\ G. J. Cuello, ${ }^{3,4}$ and F. J. Bermejo ${ }^{3,5}$ \\ ${ }^{1}$ Grup de Caracterització de Materials, Departament de Física i Enginyeria Nuclear, ETSEIB, \\ Universitat Politècnica de Catalunya, Diagonal 647, E-08028 Barcelona, Catalonia, Spain \\ ${ }^{2}$ Physik Department E13 and Forschungs-Neutronenquelle Heinz Maier-Leibnitz (FRM II), Technische Universität München, \\ Lichtenbergstr. 1, D-85748 Garching, Germany \\ ${ }^{3}$ Facultad de Ciencia y Tecnología, Universidad del País Vasco / EHU, P. Box 644, E-48080 Bilbao, Spain \\ ${ }^{4}$ Institut Laue Langevin, 6 Rue Jules Horowitz, Boîte Postale 156, F-38042 Grenoble Cedex 9, France \\ ${ }^{5}$ Instituto de Estructura de la Materia, C.S.I.C., Serrano 123, E-28006 Madrid, Spain \\ (Received 20 January 2011; revised manuscript received 23 June 2011; published 18 August 2011)
}

\begin{abstract}
We report on the interplay between the short-range order of molecules in the liquid phase of 1,1,2,2-tetrachloro1,2-difluoroethane and the possible molecular conformations, trans and gauche. Two complementary approaches have been used to get a comprehensive picture: analysis of neutron-diffraction data by a Bayesian fit algorithm and a molecular dynamics simulation. The results of both show that the population of trans and gauche conformers in the liquid state can only correspond to the gauche conformer being more stable than the trans conformer. Distinct conformer geometries induce distinct molecular short-range orders around them, suggesting that a deep intra- and intermolecular interaction coupling is energetically favoring one of the conformers by reducing the total molecular free energy.
\end{abstract}

DOI: 10.1103/PhysRevB.84.064202

PACS number(s): 61.25.Em, 61.05.F-, 33.15.Dj, 61.20.Ja

\section{INTRODUCTION}

The structure of molecular liquids has been extensively investigated by both diffraction and simulation techniques in the last few decades. The evergreen question is the relation between the intra- and intermolecular parts of the structure. In this context, one of the most studied systems has been $\mathrm{CCl}_{4}$, whose intramolecular structure has been well known for a long time. ${ }^{1,2}$ In a recent study, partial pair-distribution functions have shown that the intra- and intermolecular characteristic distances are well separated from each other. ${ }^{3}$ However, such a clear decoupling occurs only in a minority of molecular systems; even for molecular liquids with one molecular center, like $\mathrm{SbCl}_{5}$ and $\mathrm{WCl}_{6}$, the interplay between intraand intermolecular contributions can be considerable. ${ }^{4}$ The structure of liquid water, arguably the most important of all molecular liquids, has also been widely studied in this respect. Both the decoupled ${ }^{5}$ and the coupled ${ }^{6}$ approaches have been applied in structural modeling applications. In more complex systems, such as alcohols, ${ }^{7,8}$ the intramolecular structure has been proven to have an effect on the intermolecular structure at short and even intermediate distances. ${ }^{9}$

The determination of the intramolecular structure in the liquid or the solid phases is not an easy task. First, this is because the diffraction experiments used for its determination allow access to information only in reciprocal space, and, second, because any attempt to perform a fit has to deal with the strong correlations between intramolecular parameters, making it difficult to find the best fit to the data. ${ }^{9,10}$ These difficulties have been summarized in a recent work ${ }^{11}$ where a modification of the Levenberg-Marquardt algorithm is presented. In this paper we present a different approach to the problem based on Bayes theorem ${ }^{12}$ that helps to make nonlinear fits less challenging. ${ }^{13}$
The case of 1,1,2,2-tetrachloro-1,2-difluoroethane (hereafter F-112) is particularly interesting due to the fact that this compound has two rotamers and its liquid phase is a mixture of different intramolecular geometries.

Figure 1 shows the intramolecular structure of the two possible F-112 conformers, trans and gauche. As there are two degenerate possibilities for a gauche conformation but only one for a trans conformation, the fraction of molecules in the gauche state would be $2 / 3$ if there were no noticeable energy difference between the gauche and trans conformers. The structure has been determined at $0 \mathrm{~K}$ in the vacuum, i. e., without taking intermolecular interactions into account, through an ab initio calculation using the program HYPERCHEM ${ }^{14}$ with the $6-31 \mathrm{G}^{* *}$ basis set (see Table I). The atoms are arranged in staggered conformation and other dihedral angles exist only in transition states between the conformers. The $a b$ initio calculation has yielded a very similar energy for both conformers with a difference $\Delta H=0.006 \mathrm{eV}$, with gauche being slightly more stable.

The energy barrier $\left(\Delta H^{*}\right)$ and energy difference between the two conformers $(\Delta H)$ were experimentally determined using NMR, Raman and far infrared spectroscopy, and specific heat measurements, which yielded $\Delta H^{*}=0.3-0.42 \mathrm{eV}$ and $\Delta H=0.005-0.008 \mathrm{eV}^{15-19}$ This means that at $310 \mathrm{~K}$ $\left(k_{B} T \approx 0.027 \mathrm{eV}\right)$, hardly any molecule can be found in a transition state, and the gauche and trans conformers are nearly equally populated — with a slightly higher number of the lowest energy conformer.

Usually, trans rotamers are favored over gauche in the gas state of most substances because atoms in the latter have a higher steric strain due to being closer to each other. Nonetheless, several compounds have been reported to display the opposite behavior, with gauche being more stable, which has been called the "gauche effect." ${ }^{20,21}$ In particular, 

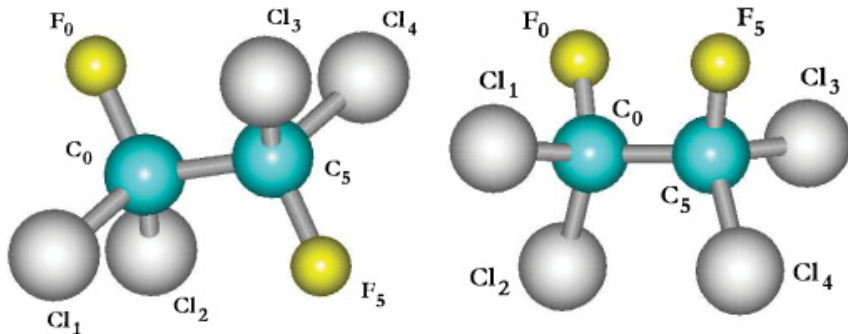

FIG. 1. (Color online) Ab initio calculation of the F-112 molecular structure in the vacuum for the trans (left) and gauche (right) conformers, together with the name convention of the atoms used in the present work.

this effect was already reported a long time ago in some halogenoethanes. ${ }^{22,23}$

The F-112 NMR spectra were measured for a 33\% and a $10 \%$ solution, and the trans conformer was found to be at a lower energy than the gauche. ${ }^{15,16}$ However, energy differences between rotational isomers strongly depend on the dielectric constant of the medium due to interactions between the polar groups of the molecule and the solvent. Therefore, these results cannot be simply applied to the case of the pure compound. ${ }^{24,25}$ Most references simply assumed the trans rotamer to be the most stable conformer, but a previous calculation of the intramolecular energy of the rotamer geometries in the vacuum, where the difference was $\Delta H=0.003 \mathrm{eV}$, returned a gauche conformer slightly more stable than the trans. ${ }^{23}$

The intramolecular structure of F-112 has previously been assumed to be decoupled from the ordering of molecules at short distances; in some molecular dynamics simulations, the molecules are even assumed to be rigid. ${ }^{26}$ Nevertheless, studies of the structure at the inter- and intramolecular scale showed that the interplay of features at these two different length scales may explain the complex dynamics of F-112. ${ }^{27-29}$

The present work is focused on the determination of the intramolecular structure of F-112, its interplay with intermolecular ordering in the liquid phase, and a deep analysis of the short-range order, and is organized as follows: After a short description of the experimental and simulation procedures, a fit method to obtain molecular parameters from the neutron diffraction data is presented. The intramolecular structure of F-112 will then be obtained from experimental data. Finally, in order to find out its relation with intermolecular length scale features, the short-range ordering of molecules will be determined with molecular dynamics simulation results using flexible F-112 molecules.

In the appendices, the error calculations when using the Fourier transform are explicitly explained, the proposed method is validated by fitting artificially generated data of the structure of an ethene molecule, and the F-112 analysis is crosschecked by fitting the molecular dynamics results and comparing the intramolecular parameters with those obtained from the experimental fit.

\section{EXPERIMENTAL AND SIMULATION DETAILS}

\section{A. Molecular dynamics simulation}

For the molecular dynamics simulations, the initial molecular geometry for F-112 was obtained by optimizing the molecular geometry at the $\mathrm{HF} / 6-31+\mathrm{G}^{*}$ level using the GAUSSIAN 03 program. ${ }^{30}$ Because a generalized AMBER force field $^{31}$ was used in the simulation to describe the intraand intermolecular interactions of $\mathrm{F}-112$, which provides a completely flexible molecular model, the molecules could change between the trans and gauche conformers to reach the equilibrium population. After assuring that consistent results were obtained for the simulation irrespective of the initial molecular conformations, a gauche conformation was adopted as the initial molecular geometry for all the molecules in the simulation. The charges used in the simulation were obtained as best fit to the molecular electrostatic potential in a certain number of points around the molecule using the CHELPG procedure. $^{32}$

The simulations were carried out for liquid F-112 using the SANDER module in the AMBER8 software package. ${ }^{33}$ The simulation box size was on average $78.5 \times 79.7 \times 77.02 \AA^{3}$ containing 2345 molecules of F-112. The temperature of the isothermal-isobaric ensemble was maintained at $310 \mathrm{~K}$ and the pressure was set to 1 bar using the Nose-Hoover and the Parrinello-Rahman algorithms, respectively. ${ }^{34}$ Once stabilization was reached, the density within the simulation box varied between 1.64 and $1.65 \mathrm{~g} / \mathrm{cm}^{3}$, which is very close to the experimental density of $1.62 \mathrm{~g} / \mathrm{cm}^{3} .{ }^{35}$ The time step for integrating the equations of motion was $1 \mathrm{fs}$ and the total time scale of the run was $10 \mathrm{~ns}$.

\section{B. Neutron diffraction experiments}

Diffraction patterns of a F-112 sample with $99 \%$ purity (ABCR GmbH \& Co. KG, Karlsruhe, Germany) were measured in the liquid phase at $310 \mathrm{~K}$. Experiments were performed at the liquids and glasses neutron diffractometer D4c at the Institute Laue-Langevin (Grenoble, France) ${ }^{36}$ using a wavelength of $\lambda=0.5 \AA$ and an angular range for the detectors that yielded a scattering vector up to $q_{\max } \approx 23 \AA^{-1}$. In order to correct and normalize the data, the empty cryostat, an empty sample holder, a boron powder sample, and a vanadium rod were also measured, as in previous works. ${ }^{37,38}$ Absorption and multiple-scattering corrections and normalization of the data were performed using the program CORRECT. ${ }^{39}$ Additionally, inelastic corrections were also carried out by subtracting a polynomial expansion in powers of $q^{2} \cdot{ }^{40}$

\section{EVALUATION OF THE DIFFRACTION DATA}

\section{A. Bayesian fit method}

Bayesian methods are routinely used in many branches of science. ${ }^{41}$ Their use in condensed matter, although it has been proven to be very powerful, is still quite scarce. ${ }^{42,43}$ Among them, the atomic reverse Monte Carlo method ${ }^{44,45}$ provides a maximum entropy solution to the problem of finding a molecular configuration that fits diffraction patterns within their errors. This method has been successfully used in the study of simple molecules such as water ${ }^{6}$ or tetrahedral molecules. ${ }^{3,37}$ However, usually a careful preparation of the initial configuration is needed in order to be able to reproduce the experimental results; a preparation that if not properly done can be misleading in the case of complex molecules such as polyalcohols. $^{7}$ 
This problem of a strong dependence of the result on the initial choice of parameters is typical for fits with many parameters. In the case of reverse Monte Carlo, the number of degrees of freedom of the system is extremely high because the description of the data is done by molecular configurations. To avoid this problem, we have developed an alternative Bayesian fit method to extract only the intramolecular structure from diffraction data. In this way, the number of parameters to describe a F-112 flexible molecule is reduced to only 23 (see below for the description of the parameters).

The classical approach to fit models to data sets is finding the parameters $\left\{P_{i}\right\}$ that minimize the figure of merit $\chi^{2}$. In contrast, Bayesian methods allow successive sets of parameters $\left\{P_{i}^{l+1}\right\}$, where $l$ is a given step in the fit process, to be accepted with a probability

$$
\mathcal{P}_{\text {accept }}=\exp \left[\frac{\chi^{2}\left\{P_{i}^{l+1}\right\}-\chi^{2}\left\{P_{i}^{l}\right\}}{2}\right] \text {. }
$$

This algorithm accepts parameters that do not decrease $\chi^{2}$ if they are consistent with the data error bars (see Appendix A). From a geometrical point of view, the parameter values are allowed to go "uphill" in the $\chi^{2}\left\{P_{i}\right\}$ hypersurface. This method has two main consequences: (i) The algorithm does not get stuck in local minima of the parameter space $\left\{P_{i}\right\}$ during the fit process. This is why the initial choice of parameter values does not matter with this fit method. ${ }^{46}$ (ii) The results are described by means of probability distribution functions (PDFs) that reflect all of the complexity of the problem under study. This is because once the best fit is found, the program explores the whole parameter space compatible with the data and its errors. Note that in the following sections, the word "parameter" will often be used in short for "parameter PDF."

\section{B. Fit function}

Independently from the choice of a Levenberg-Marquardt or a Bayesian approach to determine the best parameter set to describe the experimental data, a function has to be formulated indicating that the algorithm should fit to the data. In our case, we have applied the Bayesian method to find molecular structures compatible with a diffraction pattern-simultaneously in reciprocal and in direct space (see Appendix B 1). This procedure helps to select the best set of parameters by optimizing the path used to approach the minimum in the $\chi^{2}$ hypersurface (see Appendix B 1).

With respect to reciprocal space, in neutron diffraction of a molecular liquid, the differential scattering cross section per atom can be separated into the $q$-dependent coherent contribution from different atomic sites and a constant term arising from other contributions. ${ }^{47}$ Because the fit is intended to extract the intramolecular structure, in reciprocal space we will focus only on the intramolecular contribution of the aforementioned $q$-dependent term as performed previously, ${ }^{8-10,48,49}$

$$
\frac{1}{N}\left[\frac{d \sigma}{d \Omega}(q)\right]_{\mathrm{coh}}^{\mathrm{intra}}=\frac{1}{m} \sum_{i, j \neq i}^{m} \bar{b}_{i} \bar{b}_{j}^{*} \frac{\sin \left(q r_{i j}\right)}{\left(q r_{i j}\right)} \exp \left[-\left\langle\delta r_{i j}^{2}\right\rangle q^{2} / 2\right],
$$

where $N$ is the total number of atoms in the sample; $i$ and $j$ are sites on the same molecule; $m$ is the number of molecular sites; $\bar{b}_{i}$ is the coherent scattering length of the chemical species at site $i ; r_{i j}$ is the modulus of the mean separation between atoms $i$ and $j$; and in the Debye-Waller term, $\left\langle\delta r_{i j}^{2}\right\rangle=\left\langle u_{i}^{2}\right\rangle+$ $\left\langle u_{j}^{2}\right\rangle$, where $\left\langle u_{k}^{2}\right\rangle(k=i, j)$ is the mean-squared vibrational amplitude for the atom at site $k .^{47}$

In the case of real space, a radial distribution function $\operatorname{RDF}(r) \equiv 4 \pi r^{2} \rho g(r)$ is used, as defined in Ref. 47, derived from a Taylor-series expansion of the distances through the quadratic terms of the displacements. ${ }^{50-54}$ For distances compatible with those of the intramolecular structure, ${ }^{55}$

$$
\mathrm{RDF}_{\text {calc }}(r)=P(r)+\frac{1}{B} \sum_{i, j \neq i}^{m} \bar{b}_{i} \bar{b}_{j}^{*} \frac{r}{r_{i j}} \frac{\exp \left[-\frac{1}{2} \frac{\left(r-r_{i j}\right)^{2}}{\left\langle\delta r_{i j}^{2}\right\rangle}\right]}{\sqrt{2 \pi\left\langle\delta r_{i j}^{2}\right\rangle}},
$$

where $B=\frac{1}{m} \sum_{i, j \neq i}^{m} \bar{b}_{i} \bar{b}_{j}^{*}$ is a normalization constant, and $P(r)$ is a polynomial accounting for the density term $\left(4 \pi \rho r^{2}\right)$ and any intermolecular contribution that is visible at the intramolecular length scale. The introduction of this polynomial term is justified by the fact that the width of the first peak describing the intermolecular structure $(\approx 2-3 \AA)$ is at least an order of magnitude wider than those contributing to the intramolecular structure (between 0.01 and $0.1 \AA$ ), i.e., the peaks arising from the intramolecular structure are much sharper than the first one describing short-range order (see, for example, Refs. 37 and 7).

Because changing the relative position of a single atom within the molecule will affect all interatomic distances $r_{i j}$ related to that atom, those parameters have been reduced to a minimum set of independent variables, and since the fits are also performed taking into account molecular symmetries, the $m(m-1)$ parameters that would describe the molecular structure have been drastically diminished.

In order to obtain the experimental radial distribution function (RDF) from diffraction data, one needs to perform a Fourier transform of the measured scattering function (SF) to go from reciprocal space to real space. Unfortunately, the Fourier transformation of experimental data has two main, well-known, undesired effects in the RDF, which are due to the finite, experimentally available $q$ range: the peaks described by Eq. (3) are broadened, and a series of spurious peaks appears in addition to those defining the molecular structure. The reason is that due to experimental limitations, the recorded data is not only the SF, but its product with $\Theta\left(q_{\max }-q\right)$, where $\Theta$ is the Heaviside step function representing the experimental window. Therefore, what is obtained in direct space is the convolution of the Fourier transformation of both functions, ${ }^{47}$

$$
\mathrm{RDF}_{\text {exp }}(r)=\mathrm{RDF}_{\text {calc }}(r) \otimes \Theta^{\prime}(r),
$$

where $\Theta^{\prime}(r)=\left[1-\cos \left(q_{\max } r\right)\right] / r$ is the Fourier transform of $\Theta\left(q_{\max }-q\right)$.

The spurious peaks can be minimized (but not fully corrected) by previously applying a smoothing function to the SF that approaches zero for $q=q_{\max }$, but this solution has the drawback that the peaks are even more broadened in real space, smearing out intramolecular features. ${ }^{56}$ Instead, we have carried out this convolution in real space to fully account for both effects (peak broadening and spurious peaks) 
TABLE I. Comparison of the parameters obtained from the $a b$ initio calculation of the F-112 molecule in the vacuum with the mean and standard deviation obtained from the normal distributions of the parameters found with the fits of the experimental measurements (gauche fraction $c_{g}$, interatomic dihedral angle $\alpha$ and distances $r_{i j}$, and vibrational parameters $l_{i j}$ ). Distances related to atomic bonds and atoms bonded to the same carbon have been considered common parameters to both conformers, and distances related to atoms bonded to different carbons have been separately fitted for each conformer; those corresponding exclusively to the gauche conformation have been labeled $r^{g}$. Lengths are given in $\AA$, the angle $\alpha$ in degrees, and $c_{g}$ is adimensional.

\begin{tabular}{|c|c|c|c|c|}
\hline Parameter & $A b$ initio & Fit values & Parameter & Fit values \\
\hline$c_{g}$ & 0.67 & $0.68 \pm 0.02$ & $l_{\mathrm{CF}}$ & $0.069 \pm 0.001$ \\
\hline$\alpha$ & 59.7 & $64 \pm 2$ & $l_{\mathrm{CC}}$ & $0.0829 \pm 0.0009$ \\
\hline$r_{\mathrm{C}_{0} \mathrm{~F}_{0}}$ & 1.33 & $1.361 \pm 0.001$ & $l_{\mathrm{CC}}$ & $0.27 \pm 0.02$ \\
\hline$r_{\mathrm{C}_{0} \mathrm{Cl}_{1}}$ & 1.76 & $1.7446 \pm 0.0006$ & $l_{\mathrm{ClF}}$ & $0.27 \pm 0.01$ \\
\hline$r_{\mathrm{C}_{0} \mathrm{C}_{5}}$ & 1.56 & $1.65 \pm 0.02$ & $l_{\mathrm{F} 1 \mathrm{Cl}}$ & $0.290 \pm 0.008$ \\
\hline$r_{\mathrm{F}_{0} \mathrm{Cl}_{2}}$ & 2.52 & $2.536 \pm 0.009$ & $l_{\mathrm{ClCl}}$ & $0.16 \pm 0.01$ \\
\hline$r_{\mathrm{Cl}_{1} \mathrm{Cl}_{2}}$ & 2.88 & $2.64 \pm 0.02$ & $l_{\mathrm{Cl} 1 \mathrm{C}}$ & $0.19 \pm 0.01$ \\
\hline$r_{\mathrm{C}_{5} \mathrm{Cl}_{1}}$ & 2.73 & $2.70 \pm 0.01$ & $l_{\mathrm{F} 2 \mathrm{Cl}}$ & $0.079 \pm 0.003$ \\
\hline$r_{\mathrm{C}_{5} \mathrm{~F}_{0}}^{g}$ & 2.31 & $2.269 \pm 0.006$ & $l_{\mathrm{Cl} 2 \mathrm{C}}$ & $0.13 \pm 0.01$ \\
\hline$r_{\mathrm{C}_{5} \mathrm{Cl}_{2}}^{g}$ & 2.76 & $2.93 \pm 0.02$ & $\begin{array}{l}l_{\mathrm{Cl} 2 \mathrm{C}} \\
l_{\mathrm{F} 2 \mathrm{~F}}\end{array}$ & $<0.002$ \\
\hline$r_{\mathrm{C}_{5} \mathrm{Cl}_{1}}^{g}$ & 2.73 & $2.70 \pm 0.01$ & $l_{\mathrm{F} 2 \mathrm{~F}}$ & $0.07 \pm 0.01$ \\
\hline$r_{\mathrm{Cl}_{1} \mathrm{Cl}_{2}}^{g}$ & 2.89 & $2.70 \pm 0.04$ & ${ }^{l} \mathrm{Cl} 2 \mathrm{C}$ & $0.01+0.01$ \\
\hline
\end{tabular}

of the limited reciprocal space accessible by the experiment (see Appendix B 1). 57,58

The proposed algorithm to find the structure describing the data within their errors is implemented in the free and open, publicly available program FABADA, ${ }^{59}$ and it has already successfully been used to describe the dynamics from quasielastic neutron-scattering experiments ${ }^{60,61}$ and from dielectric spectroscopy. ${ }^{62}$

\section{THE INTRAMOLECULAR STRUCTURE OF F-112}

The intramolecular structure of F-112 in the liquid phase has been determined by simultaneously fitting the experimental neutron-diffraction SF and the RDF obtained from the Fourier transform of the data to assure the robustness of the best fit (see Appendices B 1 and B 2). For simplicity, the notation $l_{i j} \equiv \sqrt{\left\langle\delta r_{i j}^{2}\right\rangle}$ has been used. The molecular parameters $r_{i j}$ and $l_{i j}$ follow the notation of the atoms in Fig. 1 and, since the two conformers have different molecular symmetries, the needed parameters are not the same. Distances related to atomic bonds and to atoms bonded to the same carbon have been considered common parameters to both conformers, and distances related to atoms bonded to different carbons have been separately fitted for each conformer (see Table I).

Due to the symmetry of the trans conformer, the dihedral angle between its atoms $\mathrm{F}-\mathrm{C}-\mathrm{C}-\mathrm{F}$ has been assumed to be a distribution around $180^{\circ}$. The parameter $\alpha$, shown in the inset of Fig. 2, has been used to fit the dihedral angle of the gauche conformer. In order to account for the fraction of the gauche and trans conformers in the liquid state, the parameter $c_{g}$ has been introduced. It gives the gauche fraction $\left(c_{g}\right)$ and the reciprocal fraction of the trans $\left(1-c_{g}\right)$ in the liquid state. Thus, the total radial distribution function can be written as

$$
\mathrm{RDF}^{\text {intra }}=\left(1-c_{g}\right)\left(\mathrm{RDF}_{t}^{\mathrm{intra}}\right)+\left(c_{g}\right)\left(\mathrm{RDF}_{g}^{\mathrm{intra}}\right)
$$

for the RDF, and, correspondingly,

$$
\mathrm{SF}^{\text {intra }}=\left(1-c_{g}\right)\left(\mathrm{SF}_{t}^{\mathrm{intra}}\right)+\left(c_{g}\right)\left(\mathrm{SF}_{g}^{\mathrm{intra}}\right)
$$

for the SF. It must be stressed that in this equation, $c_{g}$ and $1-c_{g}$ are, like all the parameters through the Bayesian method, probability distribution functions and not just scalars (see Appendix B 1).

Since we are interested in the analysis of the intramolecular part, there is no interference between the scattering contributions of the distances between atoms in different molecules, therefore, a simple addition of each conformer contribution can be performed. However, this approach is based on the assumption that the molecules are either in the gauche or in the trans conformation, and only a negligible amount is in a transition between the two. To address the point of whether the molecules are mainly present in the gauche or trans state, the probability $P_{\text {transition }}$ of finding a molecule in a transition state between the trans and gauche conformers at the highest point of the energy barrier, with respect to the

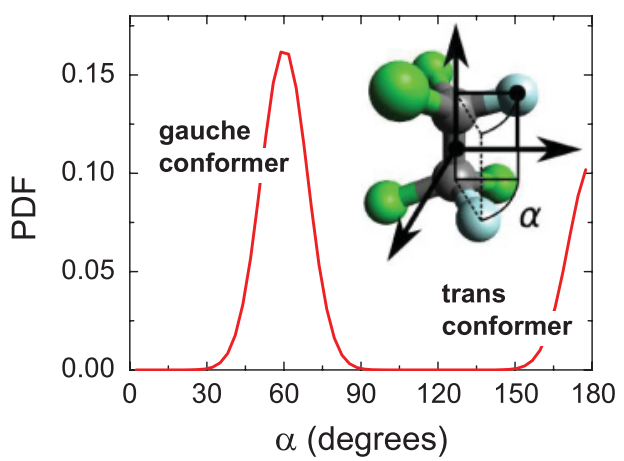

FIG. 2. (Color online) Probability distribution function (PDF) of the dihedral angle $\mathrm{F}-\mathrm{C}-\mathrm{C}-\mathrm{F}$ of the F-112 molecule as extracted from the molecular dynamics simulation in the liquid state. Inset shows the definition of the dihedral angle $\alpha$ in a F-112 molecule. 

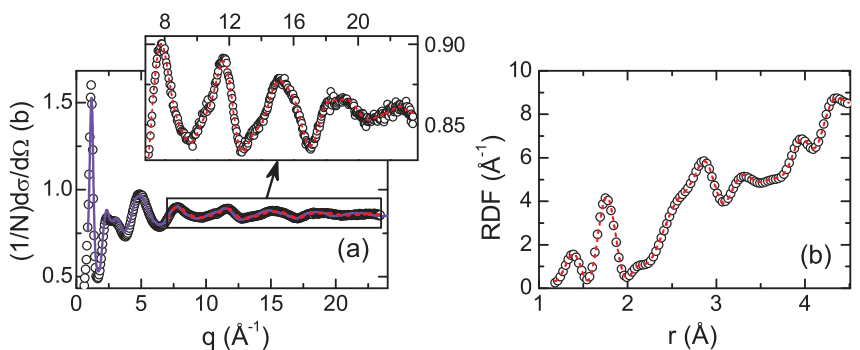

FIG. 3. (Color online) (a) Experimental SF of F-112 (circles) with its best fit (dashed orange line) in the range of higher $q$ values (here 7-23 $\AA$ ), where the intermolecular contribution is not significant, and the SF is obtained by the molecular dynamics simulation (solid violet line). The inset zooms into the fitted portion of the experimental SF, which is shown together with the best fit. (b) Experimental RDF (circles) with its best fit (dashed orange line) in the intramolecular distances domain. Note that the experimental RDF is the Fourier transform of the experimental SF and the fit is the calculated RDF convolved with $\Theta^{\prime}$.

probability $P_{\text {conf }}$ of finding it in a trans or gauche conformer, has been calculated as $P_{\text {transition }} / P_{\text {conf }}=\exp \left(\Delta H^{*} / k_{B} T\right) \approx$ $10^{-5}-10^{-7}$, when taking the literature values for $\Delta H^{*}$. We can, therefore, assess that molecules will be either in the trans or gauche conformation.

This fact is also reflected in the molecular dynamics simulation, as can be clearly seen in Fig. 2, where only the dihedral angles corresponding to trans and gauche conformers are found. The weighting method to account for the contribution of each conformer to the RDF and to the SF has been tested using data from the simulation (see Appendix B 2).

The values obtained from the ab initio simulation have been used as the initial parameters to fit the composed diffraction functions [Eqs. (5) and (6)] simultaneously in direct and reciprocal space. An excellent agreement with the experimental data is obtained for the whole experimental $q$ range (Fig. 3). The mean and standard deviations of the normal PDFs obtained from the fit of the diffraction data of the liquid phase, together with the parameters obtained from $a b$ initio calculations in the vacuum, are compiled in Table I. The molecular structure could be unambiguously determined from the experiment; of the dynamic parameters, only one related to the atomic vibrational amplitudes $\left(l_{\mathrm{F} 2 \mathrm{~F}}\right)$ could not. This is due to the small contribution that these atomic pairs have to the total intensity of the SF and the RDF (see Appendix B 1). The results summarized in Table I clearly show that the ab initio calculations (on vacuum) are substantially different.

One of the most remarkable results presented in Table I is that the obtained proportion of gauche conformer is $(68 \pm 2 \%)$. Taking into account the energy differences between trans and gauche conformers determined by previous experiments, this fraction should be between $60 \%$ and $62 \%$ at $310 \mathrm{~K}$ if the trans rotamer is assumed to be the most stable. Conversely, Fig. 4 shows that the $c_{g}$ PDF is clearly peaked at $68 \%$. In addition, this value is even higher than $2 / 3$, which is the maximal value that could theoretically be reached at high temperatures if this were the case. On the other hand, if the gauche rotamer is assumed to have the lowest energy, the gauche fraction should be between $71 \%$ and $73 \%$, and $2 / 3$ would become

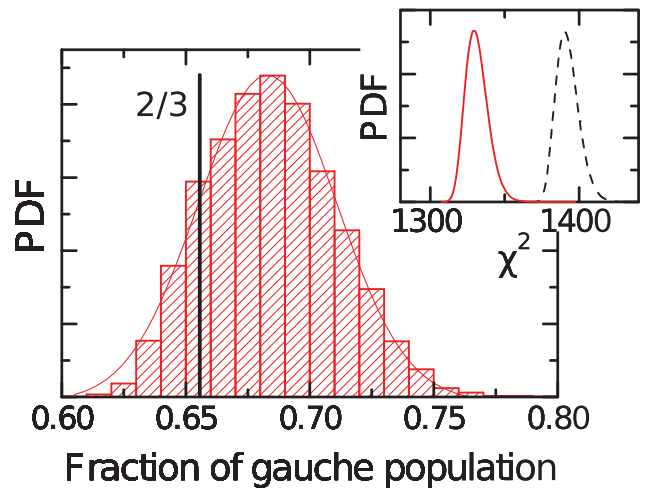

FIG. 4. (Color online) The probability density function (PDF) associated with the fraction of gauche conformer population in the sample $c_{g}$ obtained from the fit to the experimental data. Inset shows the $\chi^{2}$ PDF of hypothesis $H_{2 / 3}$ (dashed black line) and $H_{\text {free }}$ (solid red line).

the minimum theoretical value that could be reached at high temperatures, which is slightly below the actual observed value of the PDF peak.

Even disregarding the quantitative results reported in the literature for the energy difference between conformers, further support can be given to the hypothesis $H_{\text {free }}$ of having an additional parameter with any concentration $\operatorname{PDF}\left(E_{g}>E_{t}\right.$ or $E_{g}<E_{t}$, where $E_{g}$ and $E_{t}$ are the energies of the gauche and trans conformers, respectively) over the hypothesis $\mathrm{H}_{2 / 3}$ of having a fixed gauche fraction of $2 / 3\left(E_{g}=E_{t}\right)$, by comparing the $\chi^{2}$ PDFs of these two models. ${ }^{60}$ As can be seen in the inset of Fig. 4, the $H_{\text {free }}$ model is preferred to the $H_{2 / 3}$ model because the $\chi^{2}$ PDF has its peak at a smaller value, therefore disfavoring the $E_{g}=E_{t}$ scenario. As mentioned above, $E_{g}>E_{t}$ would yield a $c_{g}$ distribution below $2 / 3$, which is contrary to observation (Fig. 4), thus leading to the conclusion that in the liquid phase, $E_{g}<E_{t}$.

This analysis provides a strong indication that gauche is the more stable conformer in the F-112 liquid phase. Deviations of the gauche population from the expected values can be ascribed to possible differences in the short-range order seen by trans and gauche molecules, which could energetically favor one or the other conformer. This is analogous to the effect produced on the energy difference of the conformers by different mixtures of solvents with a distinct dielectric constant, thus coupling its intramolecular structure with the surrounding intermolecular interactions. ${ }^{24,25}$

In order to ascertain the validity of the previously stated assumption concerning the proportion of the gauche conformer due to differences in the molecular ordering, a molecular dynamics simulation using flexible molecules was performed. The simulation yields the same result that is obtained by fitting experimental data: a large fraction of gauche conformer. The value of the gauche population obtained in the simulations is $74 \%$, which is even higher than the one obtained experimentally, thus supporting the conclusion that gauche is the most stable conformer in the liquid phase. This can be qualitatively observed from the area of the peaks in Fig. 2. Last but not least, the agreement between experimental results and the simulations confers a reliable proof of the validity of the method used here (see Appendix B 2). 


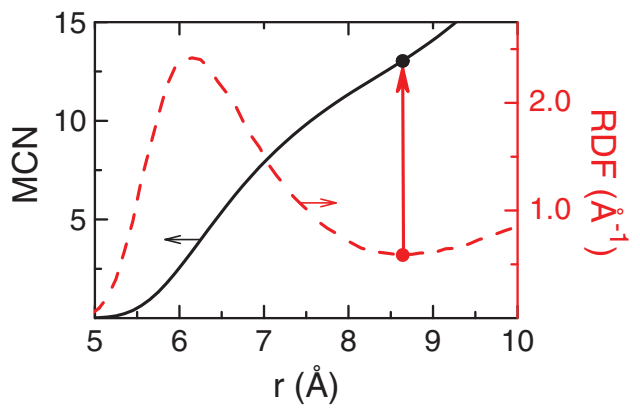

FIG. 5. (Color online) Radial distribution function (RDF) of the molecular centers of mass (dashed red line) together with the molecular coordination number (MCN) (solid black line) of F-112 from the molecular dynamics simulation. An upward arrow indicates the $\mathrm{MCN}$ value that corresponds to the RDF minimum, which was used to define the first neighbor shell. The corresponding MCN (black point) is 13 .

\section{THE INTERMOLECULAR SHORT-RANGE ORDERING OF F-112}

The first step to analyze the short-range order of F-112 is to determine the number of molecules in the first coordination shell. The molecular coordination number was obtained through the integration of the RDF of the distances between the centers of mass (with respect to a central one). The number of molecules within the first coordination shell is 13, as can be seen in Fig. 5 .

We analyze the differences between the relative positions of F-112 molecules in the first coordination shell around trans and gauche molecules. Figure 6 displays a scheme to define the angle $\phi$ used to analyze the short-range order around each conformer. For both conformers, $\phi$ is the equatorial molecular angle defined as the one between the reference molecule plane (defined by the $\mathrm{C}-\mathrm{C}$ axis and a fluorine atom) and the neighbor molecule position plane (defined by the $\mathrm{C}-\mathrm{C}$ axis of the reference molecule and the central point of the $\mathrm{C}-\mathrm{C}$ axis in the neighbor molecule).

This angle gives direct information about the arrangement of molecules. Roughly speaking, $\phi=0^{\circ}$ corresponds to configurations where neighbor molecules are in the direction of one of the fluorine atoms.

The PDF associated to the equatorial angle $\phi$ only for the first neighbor around trans and gauche conformers is depicted

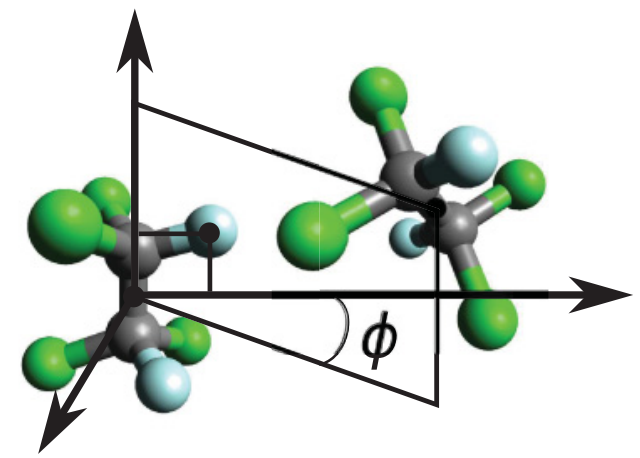

FIG. 6. (Color online) Scheme of the definition of the equatorial angle $\phi$ between the reference molecule plane and the position plane of the neighbor molecule in F-112. For both conformers, the reference molecule plane is defined by the $\mathrm{C}-\mathrm{C}$ axis and a fluorine atom, and the position plane of the neighbor molecule is defined by the $\mathrm{C}-\mathrm{C}$ axis of the reference molecule and the central point of the $\mathrm{C}-\mathrm{C}$ axis of the neighbor molecule. In the case of the trans conformer, the plane containing the other fluorine atom will be located at $\phi_{F}=180^{\circ}$, while in the case of the gauche conformer, this plane will be located at an angle $\phi_{F}=\alpha$.

in Fig. 7(a). The ordering of the molecules is different around trans and gauche conformers, as can be directly seen in the figure. Closest neighbors align preferably in the direction of the fluorine atoms, hence, due to the different geometries of both conformers, molecules tend to locate at $\phi=0^{\circ}$ and $180^{\circ}$ around trans conformers, and at $\phi=0-60^{\circ}$ around gauche conformers (in the direction of the small region between the fluorine atoms). Both profiles reflect the symmetry of its conformer geometry, which is the plane going through the $\mathrm{C}-\mathrm{C}$ axis with $\phi=0^{\circ}$ and $180^{\circ}$ for the trans, and the plane going through the $\mathrm{C}-\mathrm{C}$ axis with $\phi=30^{\circ}$ and $210^{\circ}$ for the gauche.

It is now clear that there is a difference in the ordering of the first neighboring molecules around a central trans or gauche conformer. How far does this difference reach? Figures 7(b) and 7(c) show the probability density of finding a neighbor molecule at a certain angle $\phi$ around a trans or a gauche reference molecule as a function of the molecular coordination number. This representation provides information at a first glance about the angular distribution of neighboring molecules when moving away from the reference molecule, and thus
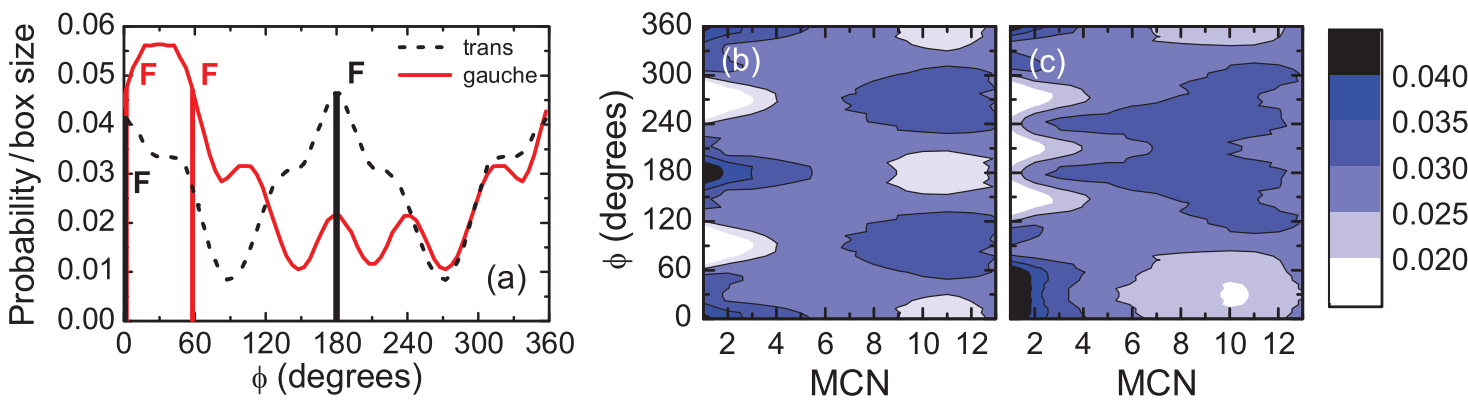

FIG. 7. (Color online) (a) Probability density of the equatorial angle of the central point $\phi$ of the first neighbor molecule $(\mathrm{MCN}=1$ ) surrounding trans (black lines) and gauche (red lines) conformers, extracted from the molecular dynamics simulation in the liquid state. Vertical lines show the direction in which the fluorine atoms are found for each conformer. The same probability density as a function of MCN surrounding (b) trans and (c) gauche conformers (darker shade means higher probability). 
gives a picture of the short-range order inside the whole first coordination shell. A look at this figure readily tells us that the relative position of molecules is different for each conformer, even at rather long distances from the reference molecule. As expected, at longer distances, the molecular ordering starts to smear out but, even then, trans and gauche molecules try to fill the gaps that the closest neighbors left empty, so a difference in the molecular arrangement is still maintained, which is a phenomenon observed as well in carbon tetrachloride. ${ }^{37}$ A complete characterization of the short-range order of F-112 using this kind of bivariate analysis ${ }^{63,64}$ is being carried out, and additional differences in the relative orientation of molecules have already been found.

\section{CONCLUDING REMARKS}

Concerning the intramolecular structure of F-112, it has been possible to show that within the liquid phase, the gauche conformer represents $(68 \pm 2 \%)$ of the molecular population, which supports the conclusion that it is the most stable conformer in the liquid phase.

The model with a distribution of an adjustable gauche conformer fraction is preferred over the model with a fixed fraction of $2 / 3$, which is a reflection that the intra- and intermolecular degrees of freedom are deeply intertwined in F-112. Thus, it is essential to use flexible molecular models that allow conformation population variability to get true insight into the behavior of this compound.

The departure from the expected conformer population has been tentatively attributed to the difference in the short-range order around trans and gauche molecules that energetically favors one of the conformers, coupling intra- and intermolecular interactions. It has been shown, through the analysis of molecular dynamics simulations, that the first neighbor molecules tend to locate in the direction of the fluorine atoms, therefore, a different molecular short-range ordering around trans and gauche molecules is induced by each conformer geometry. Angular distribution differentiates trans and gauche rotamers up to relatively long distances because even when defined positions start to blur, a contrast is still preserved since F-112 molecules fill the gaps that the closest neighbors did not occupy.

The successful use of the analysis method presented here to determine the molecular structure from diffraction data, through the fit of not only the experimentally obtained SF in the reciprocal space but also simultaneously the RDF in the real space obtained through Fourier transformation, will help to pave the way to understanding the molecular structures and the short-range order in complex disordered systems.

\section{ACKNOWLEDGMENTS}

This work was supported by the Spanish Ministry of Science and Innovation (Grant No. FIS2008-00837) and by the Government of Catalonia (Grant No. 2009SGR-1251).

\section{APPENDIX A: CALCULATING THE ERROR IN FOURIER TRANSFORMATIONS}

Errors associated with the data play a major role in the proposed fit scheme to obtain molecular structure parameters because they regulate the capability of the program to go uphill in the $\chi^{2}\left\{P_{i}\right\}$ hypersurface.

Because the method is optimized by the simultaneous fit of the functions in both the reciprocal and the direct space, it is not enough to know the experimental error from the measured SF; it is also necessary to calculate the errors of the RDF obtained after Fourier transforming the experimental data and convolving with $\Theta^{\prime}$. And since this is not an obvious calculation, it is explained in detail in this Appendix.

Errors have been calculated following two alternative approaches: by error propagation of the discretized Fourier transform and by using a Monte Carlo method. ${ }^{65}$

The discretized version of the Fourier transform that has been applied to the experimental SF to obtain the RDF is

$$
\mathrm{RDF}_{\exp }(r)=4 \pi \rho r^{2}+\frac{2 r}{\pi} \sum_{k=1}^{n} q_{k} F_{\exp }\left(q_{k}\right) \sin \left(q_{k} r\right) \Delta q,
$$

where $k=1, \ldots, n$ are the experimental points, $\Delta q$ is the sampling interval, and $F_{\text {exp }}$ is the differential scattering cross section per atom subtracted by the constant term $\bar{\sigma}_{\text {scatt }} / 4 \pi$, with $\left[\bar{\sigma}_{\text {scatt }} / 4 \pi\right]$ being the average scattering cross section. If the theory of error propagation is applied to Eq. (A1) and it is assumed that errors are normally distributed, a relationship is obtained between the errors of the SF and the RDF:

$$
\varepsilon_{\mathrm{RDF}(r)}=\varepsilon_{F(q)} \sum_{k=1}^{n} \frac{2}{\pi}\left(q_{k} r\right) \sin \left(q_{k} r\right) \Delta q .
$$
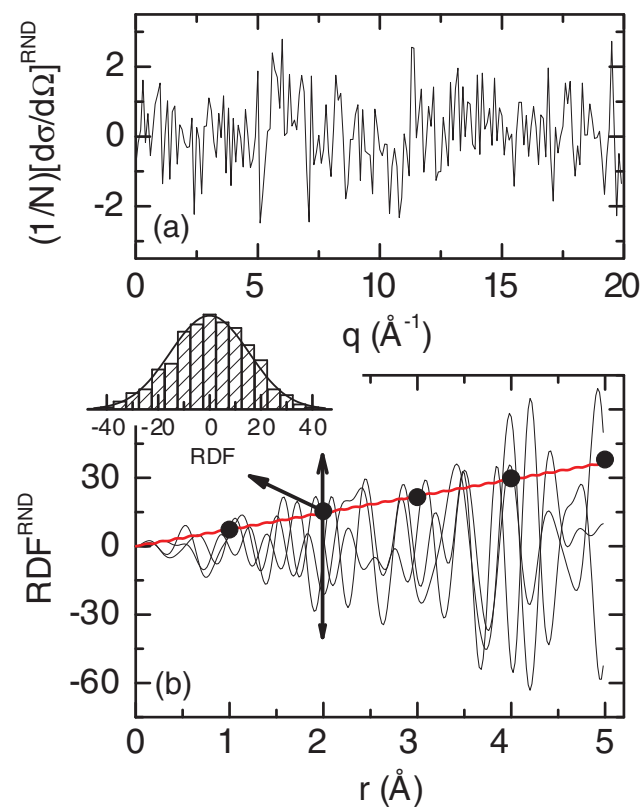

FIG. 8. (Color online) (a) Scattering function $S^{\mathrm{RND}}$ generated as normally distributed random (RND) data points. (b) Several RDF ${ }^{R N D}$ obtained from Fourier transforming different $S^{\mathrm{RND}}$ such as the one in (a) (thin black lines); errors calculated by Monte Carlo simulation (points) and by error propagation (thick red line). The inset represents the distribution of $\mathrm{RDF}^{\mathrm{RND}}$ values at a single point obtained by Fourier transforming $1000 S^{\mathrm{RND}}$, showing that data are also normally distributed in real space. 
Errors in the RDF calculated in this way have been plotted in Fig. 8(b), and are essentially linear with respect to $r$ (the slight sinusoidal modulation can barely be seen).

The Monte Carlo method to calculate errors consists of Fourier transforming SFs generated with normally distributed random points and obtaining the PDFs of the points in real space as a function of $r$ after a number of SFs have been generated and Fourier transformed in this manner. A single example of such a SF and several RDFs corresponding to Fourier transformation of different SF are shown in Figs. 8(a) and $8(\mathrm{~b})$, respectively. The PDFs of the points have been calculated, in this case, after Fourier transformation of 1000 random SFs. As can be seen in the inset of Fig. 8(b), normal distributions for the data were obtained in real space as well, validating the calculation of errors using Eq. (A2). The error at each point has been obtained from the standard deviation of its normal distribution and has been plotted in Fig. 8(b), together with the errors calculated from error propagation. As we can see in the figure, both methods lead to the same result (points from the Monte Carlo calculation lie on top of the curve from error propagation) despite the fact that the Monte Carlo method requires a considerably greater computational effort.

Using the error propagation method, a grid of the RDF error as a function of $r, \Delta q$, and $q_{\max }$ has been calculated. Figure 9 shows two series of error calculations: for fixed $\Delta q$ and for fixed $q_{\max }$ [points in Figs. 9(a) and 9(b), respectively].

From the calculated $\varepsilon\left(r, \Delta q, q_{\max }\right)$ at different $q_{\max }$ and $\Delta q$, the following relationship has been obtained:

$$
\varepsilon_{\mathrm{RDF}}(r)=m\left(q_{\mathrm{max}}, \Delta q\right) \cdot r
$$

where the value of the slope $m\left(q_{\max }, \Delta q\right)$ satisfies the empirical equation $\log _{10} m=1.5341 . \log _{10} q_{\max }+0.495 \cdot \log _{10} \Delta q-$ 0.646 . The advantage of using this equation is that since $q_{\max }$ and $\Delta q$ are usually constant parameters in diffraction experiments, $m\left(q_{\max }, \Delta q\right)$ has to be computed only once, and the error is linear with $r$.

The errors obtained using the proposed relationship have also been plotted in Fig. 9 as straight lines. Excellent agreement between both validates the proposed relationship as a convenient way to estimate errors of RDFs obtained through the Fourier transform of experimental SFs.
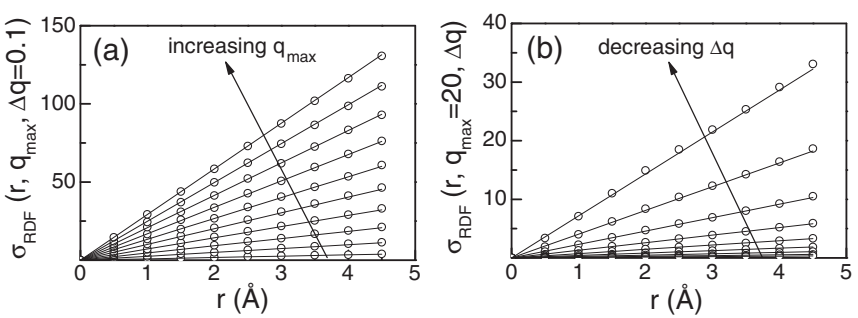

FIG. 9. Calculation of RDF errors after Fourier transformation of the SF using error propagation of random data with a normal distribution (dots) and convenient estimation of these errors through Eq. (A3) (lines). Errors have been calculated as (a) a function of $q_{\max }$, with $\Delta q$ fixed and (b) vice versa.

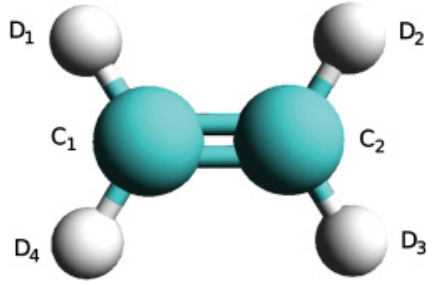

FIG. 10. (Color online) Structure of the ethene molecule used to validate the proposed algorithm, together with the name convention used in Table II.

\section{APPENDIX B: BAYESIAN FIT METHOD VALIDATION}

In order to attest the validity and robustness of the proposed fit method to obtain molecular structure parameters from diffraction data, the whole process will be performed on two known samples so that the outcome of the fit can be compared to the real values.

\section{Fitting a synthetic test molecule}

The sample used for this test consists of a generated SF data set corresponding to a deuterated ethene molecule (see Fig. 10) with a known geometry. The SF has been generated using Eq. (2) without the polynomial term $P(r)$ and adding normally distributed random numbers to those values so that the error associated with the generated experimental data set is $\sigma=0.002$. The geometric parameters used to build the molecule, listed in Table II, do not exactly correspond to the empirical ones. Once the distance between carbon atoms $r_{\mathrm{CC}}$ and between carbon and deuterium atoms $r_{\mathrm{CD}}$, and the angle between two deuterium atoms bonded to the same carbon $\alpha_{\mathrm{DCD}}$, are known, the structure of this molecule can be fully determined. Furthermore, as far as the vibrational parameters $l_{i j}$ are concerned, only six have been considered independent after taking into account the molecular symmetry.

In Fig. 11, we show the RDF of ethene obtained by Fourier transformation of the constructed experimental SF and the RDF obtained directly from the model [calculated using the seed parameters of Table II in Eq. (3) after convolving the model with $\Theta^{\prime}$ ]. Good agreement of the latter (see inset in

TABLE II. Comparison of the parameters used to generate the ethene molecular data (interatomic distances $r_{i j}$ and angle $\alpha_{i j k}$, and vibrational parameters $l_{i j}$ ) with the mean and standard deviation of the normal distributions of the parameters obtained from the fits. Lengths are in $\AA$ and angles are in degrees.

\begin{tabular}{lcc}
\hline \hline Parameter & Seed values & Fit values \\
\hline$r_{\mathrm{C}_{1} \mathrm{C}_{2}}$ & 1.3 & $1.294 \pm 0.004$ \\
$r_{\mathrm{C}_{1} \mathrm{D}_{1}}$ & 1.1 & $1.100 \pm 0.001$ \\
$\alpha_{\mathrm{DCD}}$ & 117.0 & $116.9 \pm 0.4$ \\
$l_{\mathrm{C}_{1} \mathrm{C}_{2}}$ & 0.05 & See Fig. 13 \\
$l_{\mathrm{C}_{1} \mathrm{D}_{1}}$ & 0.1 & $0.103 \pm 0.001$ \\
$l_{\mathrm{C}_{1} \mathrm{D}_{2}}$ & 0.1 & $0.098 \pm 0.003$ \\
$l_{\mathrm{D}_{1} \mathrm{D}_{4}}$ & 0.1 & $0.107 \pm 0.005$ \\
$l_{\mathrm{D}_{1} \mathrm{D}_{2}}$ & 0.2 & $0.200 \pm 0.010$ \\
$l_{\mathrm{D}_{1} \mathrm{D}_{3}}$ & 0.2 & $0.200 \pm 0.015$ \\
\hline \hline
\end{tabular}




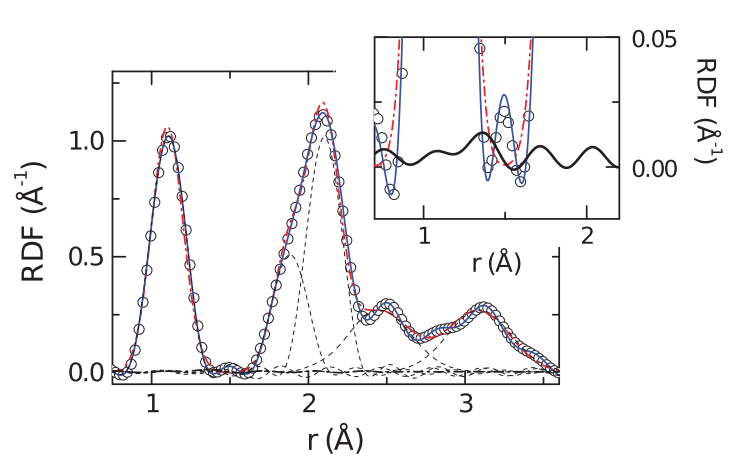

FIG. 11. (Color online) RDF of ethene obtained by Fourier transformation of the generated SF (circles), RDF calculated directly using the model (dash-dotted red line), and RDF obtained through the convolution of the model with $\Theta^{\prime}$ (solid blue line). Dashed black lines are individual contributions from the different atomic pairs (also convolved with $\Theta^{\prime}$ ). Inset zooms the part of the RDF where the small $r_{\mathrm{CC}}$ contribution is located (solid black line).

Fig. 11) supports the validity of both the Taylor approximation in Eq. (3) and the method used to account for the truncation effects in the SF.

Two ways of fitting the generated data have been undertaken to check the suitability of the proposed method: using only the $\mathrm{SF}$, and using both the SF and the RDF. As can be seen in Fig. 12(a), although both methods are able to describe the data within experimental error, the second method leads to a smaller $\chi^{2}$ value for the SF (inset of this figure). In order to clarify why fitting in both direct and reciprocal space is
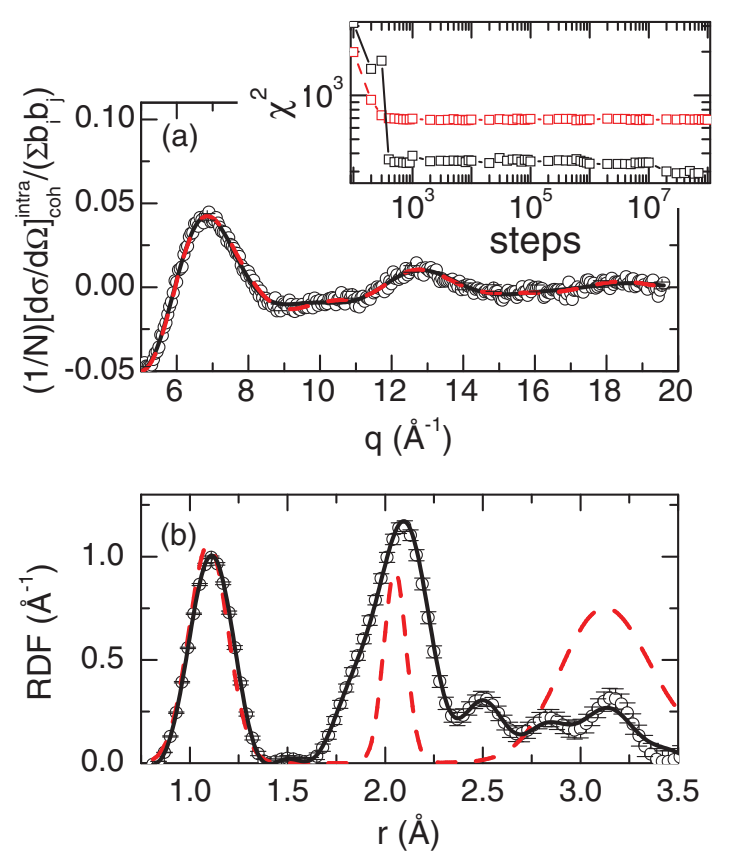

FIG. 12. (Color online) Best fits of the generated molecular structure of ethene (black circles) using only the SF (dashed red line) or using both the SF and the RDF (solid black line) of the generated data set. (a) Normalized SFs and (b) RDFs. Inset shows in semilogarithmic scale the $\chi^{2}$ evolution of the SF through the fit process using one or both functions.
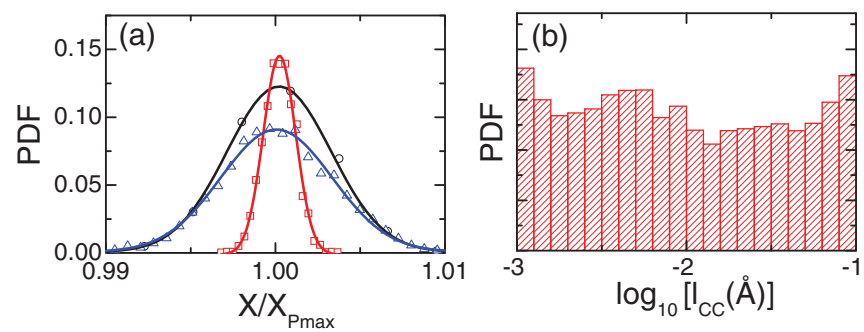

FIG. 13. (Color online) (a) PDFs obtained for the structural parameters of ethene, with the $x$ axis rescaled by the most probable value of each parameter: $r_{\mathrm{CD}}$ (red squares), $r_{\mathrm{CC}}$ (black circles), and $\alpha_{\mathrm{DCD}}$ (blue triangles). (b) PDF obtained for $l_{\mathrm{CC}}$, the vibrational parameter of the two carbon atoms of ethene.

more desirable than just in reciprocal space, we have plotted in Fig. 12(b) the RDFs obtained with the fit of only the SF and the fit where direct and reciprocal space were simultaneously used. As can readily be seen in this figure, very similar functions in reciprocal space (both of them fit the SF data within the error) may be completely different in real space, leading in some extreme cases such as this one to results devoid of physical meaning. That is because the profile of the $\chi^{2}\left\{P_{i}\right\}$ hypersurface is different in reciprocal and direct space, and, since the parameters we are using for the fit are natural real-space magnitudes, their minima appear better defined in real space. Fitting in both spaces at the same time helps thus to select the sets of parameters that are more likely to yield a closer description to the real system. Consequently, fits were performed hereafter in this way.

Figure 13 shows the PDFs as a function of rescaled parameters $\left(r_{\mathrm{CC}}, r_{\mathrm{CD}}, \alpha_{\mathrm{DCD}}\right)$ with respect to their maximum probability values. Figure 13(a) clearly evidences that PDFs are well described by normal distributions. Errors were calculated as usual in these PDFs, i.e., one standard deviation from the mean of the PDF containing $68 \%$ of values.

The same conclusion (not shown) has been obtained for the vibrational $l_{i j}$ parameters (Table II), except $l_{\mathrm{CC}}$, which could not be described at all by a normal distribution. Because not even the length scale of $l_{\mathrm{CC}}$ could be determined, a maximum-ignorance prior distribution in a logarithmic scale, or Jeffreys prior, ${ }^{66}$ has been used to calculate its PDF, which is shown in Fig. 13(b). From the figure, it is clear that it makes no sense to give a value to this parameter, because any $l_{\mathrm{CC}}$ will be able to fit both the SF and RDF equally well within the error. In order to further understand this fact, a zoom of the area in the RDF where this peak is located has been displayed in the inset of Fig. 11. The contribution of the carbon-carbon term is so small that the peak can barely be distinguished in this figure; that is the reason why $l_{\mathrm{CC}}$ cannot be determined. Nevertheless, we would like to point out that, even if this peak is not visible, since the fit is performed taking into account the whole molecular geometry, and differences in the distance between carbon atoms induce changes in the positions of many other peaks, it has been possible to accurately determine the parameter $r_{\mathrm{CC}}$ concerning the peak position of the distance between the carbon atoms' contribution. 

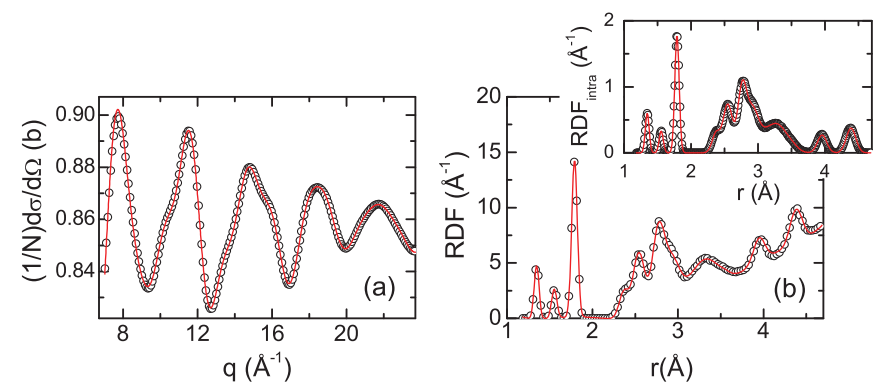

FIG. 14. (Color online) (a) Scattering function and (b) radial distribution function obtained from the molecular dynamics simulation (black circles) together with the best SF fit obtained through the proposed algorithm (red line). The inset shows the RDF intramolecular contribution of the simulation, together with the RDF calculated from the parameters of the SF best fit.

\section{Fitting the intramolecular parameters from a molecular dynamics simulation}

The mathematical procedure, i.e., the fit method and the way it has taken into account the termination effects of experimental data, has been already tested in the preceding section using a synthetic data set generated from a reasonably simple, known molecular geometry. Nevertheless, it would be useful to test the validity of the method from a data set not directly obtained from a mathematical description of a known geometry, but from a situation much closer to the experiment.

In this test, the known sample will be the SF and RDF data sets obtained from the F-112 molecular dynamics simulation. The molecular structure obtained from the SF fit will be compared to the molecular parameters that have been used in the simulation. Special attention has been devoted to test if the fit procedure is able to obtain the parameter accounting for the concentration of gauche conformer $c_{g}$; that is to say, whether the existence of two conformers with two different geometries can be accounted for by generating a function weighted by that concentration PDF [see Eqs. (5) and (6)]. In Fig. 14(a), we show the excellent agreement between the SF obtained from the simulation and its best fit obtained using the proposed algorithm. The intramolecular parameters used in the simulation and those obtained from the fit procedure are in all cases compatible with the errors.

To quantify the discrepancies between the original intramolecular structure and the parameters obtained from the fit, we introduce the relative error calculated as $\left(P_{\mathrm{MD}}-P_{\mathrm{fit}}\right) / P_{\mathrm{MD}}$, where $P_{\mathrm{MD}}$ are the parameters that have been used in the molecular dynamics simulation, and $P_{\text {fit }}$ are the parameters obtained from the fit of the SF produced by the simulation. This relative error ranges from $0.03 \%$ to $0.5 \%$ for the intramolecular distances, and from $0.6 \%$ to $13 \%$ for the vibrational parameters. As discussed in the previous section, vibrational parameters are in the first approximation uncorrelated for every atomic pair, and are thus subjected to a greater error. This error is greatly increased, even to the extreme of avoiding its determination, if the atomic-pair contribution to the total SF is small. Concerning the gauche conformer concentration, the determination from the fit has a relative error of $2 \%$, a value which lies within the $c_{g}$ error given by its resolved PDF. Therefore, we conclude that Eq. (2) correctly describes the data, and that the fit procedure is able to reproduce the original values used in the molecular dynamics simulation, including the gauche conformer fraction.

In order to verify whether Eq. (3) is a valid approximation, we have also calculated the intramolecular RDF, shown in the inset of Fig. 14, using the parameters that have been obtained through the fit of the SF. The resemblance between the intramolecular RDF calculated from the simulation and the one calculated from the SF fit is virtually perfect, thus evidencing that the approximation in Eq. (3) used to describe the RDF is appropriate. Moreover, we have also tested which is the effect of modeling the intermolecular contribution as a polynomial, as it is performed in our work with the real data. The fit obtained using this approximation is extremely good, as can be seen in Fig. 14(b). The parameters determined from this fit are again close to those used in the simulation. In this case, the relative errors of the distances range from $0.1 \%$ to $3 \%$, errors from the vibrational parameters range from $0.6 \%$ to $7 \%$, and the error in the gauche concentration is about $2 \%$, all of which are within the error bars of the fit determination.

\section{Validation summary}

The tests performed using generated ethene SF and RDF data sets, and performed on molecular dynamics data, allow us to conclude the following:

(i) We properly accounted for finite information effects of the experimental data (termination effects of the SF).

(ii) The approximation used to describe the RDF derived from a Taylor-series expansion of the distances through the quadratic terms of the displacements is valid.

(iii) The fit procedure is robust, both determining intramolecular distances and rotamer concentrations, due to the fact that these parameters are strongly interrelated. Even distances whose contribution cannot be observed either in the SF or the RDF can be determined due to their dependence on the remaining intramolecular distances.

(iv) Vibrational parameters may be determined only if their contribution is significant enough. But in any case, the obtained PDFs using the proposed method allow us, at least, to limit their values.

(v) A polynomial can be used to model the intermolecular contribution to the RDF, with the drawback of slightly increasing the associated errors of the intramolecular parameters. For the particular case of F-112, the modelization carried out to take into account the rotamer population in the calculation of the SF and the RDF has been successfully established by weighting each conformer contribution by its concentration.

\footnotetext{
${ }^{1}$ A. H. Narten, M. D. Danford, and H. A. Levy, J. Chem. Phys. 46, 4875 (1967).

${ }^{2}$ P. A. Egelstaff, D. I. Page, and J. G. Powles, Mol. Phys. 20, 881 (1971).
}

\footnotetext{
${ }^{3}$ Sz. Pothoczki, L. Temleitner, P. Jovari, S. Kohara, and L. Pusztai, J. Chem. Phys. 130, 064503 (2009).

${ }^{4}$ L. Pusztai and R. L. McGreevy, J. Chem. Phys. 125, 044508 (2006).

${ }^{5}$ A. K. Soper, J. Phys. Condens. Matter 22, 404210 (2010).
} 
${ }^{6}$ L. Pusztai, Phys. Rev. B 60, 11851 (1999).

${ }^{7}$ A. Vrhovsek, O. Gereben, Sz. Pothoczki, M. Tomsic, A. Jamnik, S. Kohara, and L. Pusztai, J. Phys. Condens. Matter 22, 404214 (2010).

${ }^{8}$ A. Adya, L. Bianchi, and C. Wormald, J. Chem. Phys. 112, 4231 (2000).

${ }^{9}$ C. Talón, F. J. Bermejo, C. Cabrillo, G. J. Cuello, M. A. González, J. W. Richardson, A. Criado, M. A. Ramos, S. Vieira, F. L. Cumbrera, and L. M. González, Phys. Rev. Lett. 88, 115506 (2002).

${ }^{10}$ G. Venturi, F. Formisano, G. J. Cuello, M. R. Johnson, E. Pellegrini, U. Bafile, and E. Guarini, J. Chem. Phys. 131, 034508 (2009).

${ }^{11}$ M. K. Transtrum, B. B. Machta, and J. P. Sethna, Phys. Rev. Lett. 104, 060201 (2010).

${ }^{12}$ T. Bayes and R. Price, Philos. Trans. Roy. Soc. London 53, 370 (1763).

${ }^{13}$ L. C. Pardo, M. Rovira-Esteva, S. Busch, M. D. Ruiz-Martín, J. Ll. Tamarit, and T. Unruh, e-print arXiv:0907.3711 (to be published).

${ }^{14}$ Computer code HYPERCHEM PROFESSIONAL 7.51 (Hypercube, Inc., Gainesville, FL, 2003).

${ }^{15}$ R. A. Newmark and C. H. Sederhol, J. Chem. Phys. 43, 602 (1965).

${ }^{16}$ R. A. Newmark and R. E. Graves, J. Phys. Chem. 72, 4299 (1968).

${ }^{17}$ R. E. Kagarise and L. W. Daasch, J. Chem. Phys. 23, 113 (1955).

${ }^{18}$ R. A. Pethrick and E. Wynjones, J. Chem. Soc. A 54 (1971).

${ }^{19}$ K. Kishimoto, H. Suga, and S. Seki, Bull. Chem. Soc. Jpn. 51, 1691 (1978).

${ }^{20}$ S. Wolfe, Acc. Chem. Res. 5, 102 (1972).

${ }^{21}$ R. C. Bingham, J. Am. Chem. Soc. 98, 535 (1976).

${ }^{22}$ J. R. Durig, J. Liu, T. S. Little, and V. F. Kalasinsky, J. Phys. Chem. 96, 8224 (1992).

${ }^{23}$ R. J. Abraham and K. Parry, J. Chem. Soc. B 539 (1970).

${ }^{24}$ R. J. Abraham and M. A. Cooper, Chem. Commun. 588 (1966).

${ }^{25}$ R. J. Abraham and M. A. Cooper, J. Chem. Soc. B 202 (1967).

${ }^{26}$ F. Affouard, E. Cochin, F. Danede, R. Decressain, M. Descamps, and W. Haeussler, J. Chem. Phys. 123, 084501 (2005).

${ }^{27}$ L. C. Pardo, P. Lunkenheimer, and A. Loidl, J. Chem. Phys. 124, 124911 (2006).

${ }^{28}$ L. C. Pardo, F. J. Bermejo, J. Ll. Tamarit, G. J. Cuello, P. Lunkenheimer, and A. Loidl, J. Non-Cryst. Solids 353, 999 (2007).

${ }^{29}$ M. Rovira-Esteva, L. C. Pardo, J. Ll. Tamarit, and F. J. Bermejo, in Metastable Systems under Pressure, edited by S. J. Rzoska, A. Drozd-Rzoska, and V. Mazur, NATO Science for Peace and Security Series: A. Chemistry and Biology (Springer, Netherlands, 2009), pp. 63-77.

${ }^{30}$ M. J. Frisch, G. W. Trucks, H. B. Schlegel, G. E. Scuseria, M. A. Robb, J. R. Cheeseman, J. J. A. Montgomery, T. Vreven, K. N. Kudin, J. C. Burant, J. M. Millam, S. S. Iyengar, J. Tomasi, V. Barone, B. Mennucci, M. Cossi, G. Scalmani, N. Rega, G. A. Petersson, H. Nakatsuji, M. Hada, M. Ehara, K. Toyota, R. Fukuda, J. Hasegawa, M. Ishida, T. Nakajima, Y. Honda, O. Kitao, H. Nakai, M. Klene, X. Li, J. E. Knox, H. P. Hratchian, J. B. Cross, V. Bakken, C. Adamo, J. Jaramillo, R. Gomperts, R. E. Stratmann, O. Yazyev, A. J. Austin, R. Cammi, C. Pomelli, J. W. Ochterski, P. Y. Ayala, K. Morokuma, G. A. Voth, P. Salvador, J. J. Dannenberg, V. G. Zakrzewski, S. Dapprich, A. D. Daniels, M. C. Strain, O. Farkas, D. K. Malick, A. D. Rabuck, K. Raghavachari, J. B. Foresman, J. V. Ortiz, Q. Cui, A. G. Baboul, S. Clifford, J. Cioslowski, B. B. Stefanov, G. Liu, A. Liashenko, P. Piskorz, I. Komaromi, R. L. Martin, D. J. Fox, T. Keith, M. A. Al-Laham, C. Y. Peng, A. Nanayakkara, M. Challacombe, P. M. W. Gill, B. Johnson,
W. Chen, M. W. Wong, C. Gonzalez, and J. A. Pople, computer code GAUSSIAN 03, REVISION B.05 (Gaussian, Inc., Pittsburgh, PA, 2003).

${ }^{31}$ J. M. Wang, R. M. Wolf, J. W. Caldwell, P. A. Kollman, and D. A. Case, J. Comput. Chem. 25, 1157 (2004).

${ }^{32}$ C. M. Breneman and K. B. Wiberg, J. Comput. Chem. 11, 361 (1990).

${ }^{33}$ D. A. Case, I. T. E. Cheatham, C. L. Simmerling, J. Wang, R. E. Duke, R. Luo, K. M. Merz, B. Wang, D. A. Pearlman, M. Crowley, S. Brozell, V. Tsui, H. Gohlke, J. Mongan, V. Hornak, G. Cui, P. Beroza, C. Schafmeister, J. W. Caldwell, W. S. Ross, and P. A. Kollman, computer code AMBER 8 (University of California, San Francisco, CA, 2004).

${ }^{34}$ M. Parrinello and A. Rahman, Phys. Rev. Lett. 45, 1196 (1980).

${ }^{35}$ F. Hovorka and F. E. Geiger, J. Am. Chem. Soc. 55, 4759 (1933).

${ }^{36}$ H. E. Fischer, G. J. Cuello, P. Palleau, D. Feltin, A. C. Barnes, Y. S. Badyal, and J. M. Simonson, Appl. Phys. A 74, S160 (2002).

${ }^{37}$ L. C. Pardo, J. Ll. Tamarit, N. Veglio, F. J. Bermejo, and G. J. Cuello, Phys. Rev. B 76, 134203 (2007).

${ }^{38}$ G. J. Cuello, J. Phys. Condens. Matter 20, 244109 (2008).

${ }^{39} \mathrm{M}$. Howe, R. McGreevy, and P. Zetterström, computer code CORRECT (NFL Studsvik internal report, 1996).

${ }^{40}$ G. Placzek, Phys. Rev. 86, 377 (1952).

${ }^{41}$ R. Trotta, Contemp. Phys. 49, 71 (2008).

${ }^{42}$ D. S. Sivia, C. J. Carlile, W. S. Howells, and S. Konig, Physica B 182, 341 (1992).

${ }^{43}$ S. Bacallado, J. D. Chodera, and V. Pande, J. Chem. Phys. 131, 045106 (2009).

${ }^{44}$ R. L. McGreevy, J. Phys. Condens. Matter 13, R877 (2001).

${ }^{45}$ R. L. McGreevy and L. Pusztai, Mol. Simul. 1, 359 (1988).

${ }^{46}$ L. C. Pardo, M. Rovira-Esteva, S. Busch, J.-F. Moulin, and J. L1. Tamarit (unpublished).

${ }^{47}$ H. E. Fischer, A. C. Barnes, and P. S. Salmon, Rep. Prog. Phys. 69, 233 (2006).

${ }^{48}$ F. Leclercq, P. Damay, M. Founaki, P. Chieux, M. C. BellissentFunel, A. Rassat, and C. Fabre, Phys. Rev. B 48, 2748 (1993).

${ }^{49}$ M. Kreitmeir, G. Heusel, H. Bertagnolli, K. Todheide, C. J. Mundy, and G. J. Cuello, J. Chem. Phys. 122, 154511 (2005).

${ }^{50}$ R. W. James, Phys. Z. 33, 737 (1932).

${ }^{51}$ M. Iwasaki, S. Nagase, and R. Kojima, Bull. Chem. Soc. Jpn. 30, 230 (1957).

${ }^{52}$ J. Karle, Diffraction Studies on Non-crystalline Substances, edited by I. Hargittai and W. J. Orville-Thomas (Elsevier, Amsterdam, and Akadmiai Kiad, Budapest, 1981), pp. 243-267.

${ }^{53}$ M. Alvarez, F. J. Bermejo, P. Chieux, E. Enciso, J. Alonso, and N. Garcia, J. Phys. Condens. Matter 1, 8595 (1989).

${ }^{54}$ P. Rozier, A. Burian, and G. J. Cuello, J. Non-Cryst. Solids 351, 632 (2005).

${ }^{55}$ K. A. Johnson and W. S. Howells, J. Phys. Condens. Matter 11, 9239 (1999).

${ }^{56}$ E. Lorch, J. Phys. C 2, 229 (1969).

${ }^{57}$ I. Petri, P. S. Salmon, and H. E. Fischer, Phys. Rev. Lett. 84, 2413 (2000).

${ }^{58}$ J. Waser and V. Schomaker, Rev. Mod. Phys. 25, 671 (1953).

${ }^{59}$ L. C. Pardo, computer code FABADA (Barcelona, Spain, 2009); L. C. Pardo, M. Rovira-Esteva, S. Busch, M. D. Ruiz-Martin, and J. Ll. Tamarit, J. Phys. Conf. Ser. (unpublished).

${ }^{60}$ S. Busch, C. Smuda, L. C. Pardo, and T. Unruh, J. Am. Chem. Soc. 132, 3232 (2010). 
${ }^{61}$ M. Rovira-Esteva, A. Murugan, L. C. Pardo, S. Busch, M. D. RuizMartin, M. S. Appavou, J. Ll. Tamarit, C. Smuda, T. Unruh, F. J. Bermejo, G. J. Cuello, and S. J. Rzoska, Phys. Rev. B 81, 092202 (2010)

${ }^{62}$ J. C. Martinez-Garcia, J. Ll. Tamarit, L. C. Pardo, M. Barrio, S. J. Rzoska, and A. Droz-Rzoska, J. Phys. Chem. B 114, 6099 (2010).

${ }^{63}$ L. C. Pardo, M. Rovira-Esteva, J. L1. Tamarit, N. Veglio, F. J. Bermejo, and G. J. Cuello, in Metastable Systems under Pressure, edited by S. J. Rzoska, A. Drozd-Rzoska, and V. Mazur, NATO Science for Peace and Security Series: A. Chemistry and Biology (Springer, Netherlands, 2009), pp. 79-91.

${ }^{64}$ P. Jedlovszky, A. Vincze, and G. Horvai, Phys. Chem. Chem. Phys. 6, 1874 (2004).

${ }^{65}$ J. M. Fornies-Marquina, J. Letosa, M. Garcia-Gracia, and J. M. Artacho, IEEE Trans. Magn. 33, 1456 (1997).

${ }^{66} \mathrm{H}$. Jeffreys, Theory of Probability (Clarendon, Oxford, 1939). 